\title{
Ecuaciones para establecer una regresión entre las unidades de corriente con respecto a la edad del paciente y el tiempo de uso de los implantes cocleares
}

\author{
Equations to establish a regression between current units with respect to patient's age \\ and the use time of cochlear implants
}

\author{
Ileana Gutiérrez-Farfán ${ }^{1}$, Ana L. Lino-González², Antonio Verduzco-Mendoza², \\ Roberto de Leo-Vargas ${ }^{3}$ y Emilio Arch-Tirado * \\ 'Servicio de Audiología, Posgrado de Alta Especialidad de Audiología Pediátrica, Instituto Nacional de Rehabilitación Luis Guillermo Ibarra Ibarra; \\ 2Subdirección de Investigación Clínica, División de Neurociencias, Servicio de Neurociencia Clínica, Área de Discapacidad del Lenguaje, Voz y \\ Audición, Instituto Nacional de Rehabilitación Luis Guillermo Ibarra Ibarra; ${ }^{3} \mathrm{Centro}$ Neurológico del Centro Médico ABC Santa Fe; ${ }^{4} \mathrm{Campus}$ Online, \\ Universidad Tecnológica de México. Ciudad de México, México
}

\begin{abstract}
Resumen
Objetivo: Proponer un sistema de ecuaciones de regresión múltiple multivariable para establecer una asociación matemática que permita estimar las unidades de corriente necesarias durante la fase de ajuste con respecto a la edad y el tiempo de uso del implante coclear. Método: Estudio transversal descriptivo. Participaron 41 pacientes pediátricos con implante coclear unilateral. Se conformaron tres grupos y se construyeron ecuaciones de regresión múltiple multivariable. Resultados: Para el grupo 1 se observa en los grupos de electrodos 3 y 4 menor desviación estándar y similar índice de asimetría de las unidades de corriente; en el grupo 2, los grupos de electrodos con similar índice de asimetría fueron el 2 y el 4, y el grupo de electrodos 3 presentó un promedio de 21.54 y una dispersión más pequeña (16,25); en el grupo 3, los grupos de electrodos 3 y 4 presentaron similar desviación estándar y promedio y desviación estándar más pequeños. Con respecto al error estándar de estimación, se obtuvo una menor variabilidad de unidades de corriente en el grupo 2 para el grupo de electrodos 3. Conclusión: Las ecuaciones propuestas son de utilidad clínica al conocer las unidades de corriente necesarias considerando la edad y el tiempo de uso del implante coclear.
\end{abstract}

Palabras Clave: Grupo de electrodos. Implante coclear. Unidades de corriente.

\begin{abstract}
Objective: Propose a system of multivariate multiple regression equations in order to establish a mathematical association that allows estimating the current units needed during the adjustment phase with respect to age and time that were used the implants. Method: Descriptive cross-sectional study. A total of 41 pediatric patients with a unilateral cochlear implant participated. Three groups were formed and multivariable multiple regression equations were constructed. Results: For Group 1, in electrode Groups 3 and 4, there is a lower standard deviation and a similar index of asymmetry of the current units; in Group 2, the groups of electrodes with similar index of asymmetry were 2 and 4, while group of electrodes 3 presented average of 21.54 and dispersion smaller (16.25); in Group 3, groups of electrodes 3 and 4 presented similar standard deviation and aver-
\end{abstract}

Correspondencia:

*Emilio Arch-Tirado

Blvd. Calacoaya No. 7,

Col. La Ermita, Atizapán de Zaragoza, $\quad$ Fecha de recepción: 10-01-2019

C.P. 52999, Edo. de México, México Fecha de aceptación: 06-04-2019

Cir Cir. 2020;88(2):154-162

E-mail: arch.tirado@gmail.com

DOI: $10.24875 / C I R U .19001021$

Contents available at PubMed

www.cirugiaycirujanos.com

0009-7411/@ 2019 Academia Mexicana de Cirugía. Publicado por Permanyer. Este es un artículo open access bajo la licencia CC BY-NC-ND (http://creativecommons.org/licenses/by-nc-nd/4.0/). 
age and smaller standard deviation. With respect to the standard estimation error, the lowest variability of current units was obtained in Group 2 for electrode Group 3. Conclusion: The proposed equations could be used in the clinical area by knowing the units current needed with respect to age and time of use the implant.

Key Words: Electrode group. Cochlear implant. Current units.

\section{Introducción}

La Organización Mundial de la Salud reportó en 2018 una prevalencia de 466 millones de personas con discapacidad auditiva en el mundo, de las cuales 34 millones son población pediátrica'. En México, el Censo de Población y Vivienda de 2010 refirió que el $12.1 \%$ de los mexicanos con discapacidad padecían problemas auditivos ${ }^{2}$, y en 2014 la Dirección General de Análisis y Prospectiva con datos de la Encuesta Nacional de Ingresos y Gastos de los Hogares señaló que la población de personas con algún tipo de discapacidad auditiva que pretendía ingresar a algún programa público fue de 1.4 millones (18.3\%), de una población potencial de 7.7 millones $^{3}$.

El implante coclear es un dispositivo electrónico pequeño y complejo que facilita la percepción del sonido a personas con hipoacusia neurosensorial profunda o grave. En los últimos años han alcanzado popularidad, y en diciembre de 2012 aproximadamente 324,200 dispositivos fueron implantados en todo el mundo. La adaptación del implante coclear requiere un procedimiento quirúrgico y de seguimiento audiológico para garantizar su acoplamiento y óptimo funcionamiento ${ }^{4}$, alcanzado mediante una programación del dispositivo basada en ajustes periódicos de los parámetros psicofísicos y neurofisiológicos ${ }^{5}$ relacionados con la estimulación y el procesamiento de la señal ${ }^{6}$.

\section{Determinación de los umbrales}

En el proceso de programación del implante coclear es fundamental la elaboración de mapas auditivos, en los cuales se registra el campo eléctrico requerido por cada paciente con la finalidad de establecer la cantidad de microvoltios necesarios para generar la sensación auditiva ${ }^{7-10}$. Están determinados por el umbral de estimulación eléctrica o umbral T (UT), que corresponde a la cantidad mínima de corriente que produce una sensación auditiva, y el umbral de confort o umbral C/M (UC/M), correspondiente al nivel máximo de estimulación eléctrica que el paciente refiere como un sonido confortable ${ }^{5,6,11,12}$. La determinación del UT y el UC/M se basa en juicios psicofísicos de sonoridad medidos en unidades clínicas de corriente eléctrica, frecuentemente denominadas unidades de corriente (uc) 0 unidades de carga ${ }^{7-10,13,14}$.

El UC/M puede obtenerse mediante pruebas subjetivas (observación de la conducta), pruebas objetivas (respuesta neurofisiológica) o la combinación de ambas ${ }^{11,13}$. La detección de los umbrales se lleva a cabo en todos los electrodos del implante coclear, los cuales se encuentran ubicados de forma tonotópica en la cóclea representando las diferentes bandas de frecuencia (graves, medias y agudas); otros parámetros importantes considerados durante el ajuste son la velocidad, la estrategia de codificación de la señal, el ancho de pulso y el modo de estimulación $n^{13,14}$.

Deficiencias en la calibración o activación de los UC/M generan una inadecuada recepción de las señales acústicas representativas de la banda espectral asociada a los electrodos, alterando el reconocimiento de la voz, obstaculizando la calidad de sonido y limitando las habilidades de autocontrol de la voz del paciente; por otra parte, se refiere que la subestimulación o la sobreestimulación generan sonidos débiles o muy intensos, lo que provoca una percepción incómoda de las señales acústicas y dificulta el reconocimiento de la voz, afecta la calidad del sonido o produce aversión al implante ${ }^{5,15}$. Los umbrales y la cantidad de corriente eléctrica requeridos para desencadenar la sensación auditiva son distintos en cada paciente, por lo que, para cada canal, la programación del procesador del habla debe ser individualizada ${ }^{5,16}$.

\section{Unidades de corriente}

Las uc son valores numéricos utilizados para designar la cantidad de corriente liberada hacia los electrodos ${ }^{7}$,con la finalidad de determinar el umbral eléctrico de estimulación (menor cantidad de estimulación eléctrica) requerida por el paciente. La denominación de ambos parámetros varía dependiendo del fabricante del implante coclear (unidad clínica, nivel de corriente, unidad de corriente 0 unidad de carga) $)^{5,17,18}$. 


\section{Ajuste de implante coclear con métodos de valoración subjetiva}

La audiometría como técnica de medición psicoacústica puede ser un método subjetivo al generar dificultad en la obtención confiable del umbral audiológico durante la calibración del implante coclear, por factores como la edad del paciente (edad temprana), comorbilidad neurológica, conductuales o cognitivos asociados a la deficiencia auditiva ${ }^{19}$.

\section{Ajuste del implante coclear con métodos de valoración objetiva}

El reflejo estapedial eléctricamente evocado $(\text { REEE })^{20}$, la telemetría de respuesta neural, los potenciales provocados auditivos de tallo cerebral (PPATC), los potenciales provocados cognitivos, la imagen de respuesta neural y los potenciales de acción con componente eléctrico (PACE) son algunas de las herramientas clínicas objetivas que permiten determinar las uc requeridas durante el proceso de ajuste ${ }^{10,16,20-22}$.

Los PACE son los más frecuentemente utilizados en la evaluación de la actividad del nervio auditivo, la cual corresponde a la onda I en los PPATC en usuarios de implante coclear $^{23}$. El umbral de estos potenciales se encuentra en general entre los niveles del UT y del UC/M (medidos en ocasiones por las reacciones conductuales observables en los pacientes), por lo que los PACE, los PPATC y el REEE se utilizan durante la elaboración de los mapas auditivos como métodos de valoración objetiva para la obtención de estos umbrales, principalmente en pacientes pediátri$\cos ^{16,24-27}$. En el caso de los PPATC, la amplitud de la onda $V$ se incrementa al aumentar los niveles de estimulación ${ }^{28}$. Walkowiak, et al. ${ }^{27}$ y Kamal ${ }^{7}$ reportaron que existe una fuerte correlación entre el REEE y los UC/M para cada electrodo. El coeficiente de correlación entre el UC/M y el REEE es significativamente mayor $(r=0.75)$; en caso contrario, la correlación entre el UC/M y los PACE el valor era $r=0.39$.

\section{EI REEE en el ajuste del implante coclear}

Diversos estudios reportan que el REEE es una prueba electrofisiológica que debe considerarse en el proceso de activación del implante coclear y para la elaboración de los mapas auditivos, al incidir de forma positiva en aspectos de tiempo, calidad y bienes-

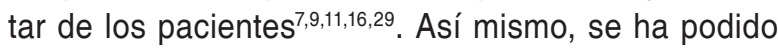

establecer que este estudio permite determinar el adecuado funcionamiento del implante coclear, así como de la vía auditiva y su conexión con los núcleos motores homolateral y contralateral del VIII par craneal a nivel de tallo cerebral ${ }^{20,30}$.Lorens, et al. ${ }^{31}$ demostraron que la programación del implante coclear basada en la respuesta del REEE para obtener UC/M da mejores resultados que la programación realizada con pruebas conductuales.

Raghunandhan, et al. ${ }^{10}$ analizaron los umbrales de los electrodos para obtener el REEE y observaron un incremento gradual ascendente, desde los dispuestos en la región apical (frecuencias graves) hasta los localizados en la región basal (frecuencias agudas); por consiguiente, lo mismo se observó en los valores de los UC/M, con un promedio de 36.3 uc ( \pm 7 desviaciones estándar) entre ambas regiones (apical y basal) en pacientes con experiencia de 1 año de uso del implante coclear. Los UC/M más elevados se ubicaron en la región basal de la cóclea, lo que implicó la necesidad de impulsos más intensos para los electrodos en esta región, la cual se caracteriza por tener una alta densidad de ganglios espirales que codifican para altas frecuencias de estimulación auditiva. Así mismo, se ha reportado que existe una tendencia de incremento en los UC/M a lo largo del tiempo, con un ascenso gradual que inicia con un promedio de 155 uc en el primer mes de uso y llega hasta 272 uc al final del primer año de la implantación. Cuando se establece un mapa auditivo inicial basado en el REEE en un paciente pediátrico no cooperador hay que ser cauteloso para evitar cualquier nivel de mapeo por encima de los umbrales, ya que puede inducir una respuesta incómoda por la sobreestimulación acústica ${ }^{10}$.

El objetivo del presente trabajo es proponer un sistema de ecuaciones de regresión múltiple multivariable con la finalidad de establecer una asociación matemática que permita estimar las uc necesarias durante la fase de ajuste con respecto a la edad del paciente y el tiempo de uso del implante coclear.

\section{Método}

Estudio transversal descriptivo realizado en el departamento de audiología pediátrica de un hospital de alta especialidad con sede en la Ciudad de México. Participaron 41 pacientes con edades comprendidas entre 2 años y 1 mes y 7 años y 6 meses con implante coclear unilateral Advanced Bionics ${ }^{\circledR}$ y un tiempo mínimo de uso de 6 meses. El protocolo fue aprobado 
por el Comité de Investigación y Ética. Todos los padres o tutores, después de la explicación de las intervenciones, firmaron un consentimiento informado de acuerdo con la Declaración de Helsinki, y se contó con el asentimiento informado de cada uno de los pacientes.

\section{Obtención del REEE}

Se utilizó el software Advanced Bionics Sound Wave ${ }^{\circledR}$ versión 2.2. Se empleó estimulación Speech Burst para obtener el REEE del oído contralateral al implantado, valorando los 16 electrodos del implante coclear divididos en cuatro grupos: el primer grupo (GE1) incluye los electrodos 1 al 4; el segundo grupo (GE2), los electrodos 5 a 8; el tercer grupo (GE3), los electrodos 9 a 12; y el cuarto grupo (GE4), los electrodos 13 a 16.

El UC/M fue el parámetro de referencia para estimar las uc necesarias para alcanzar el REEE; cuando no había respuesta, las uc se fueron incrementando paulatinamente en unidades de 5 hasta lograr identificarlo (en caso de molestia, el incremento de uc se realizaba de 1 a $1 \mathrm{~dB}$ ). Durante la prueba, el implante coclear permaneció encendido para probar clínicamente la tolerancia a viva voz (Live-Speech). El REEE obtenido se corroboró en tres ocasiones.

\section{Análisis estadístico}

Para el análisis se formaron tres grupos: el grupo 1, conformado con el total de la muestra ( $n=41$ ), $16(39 \%)$ niños y $25(61 \%)$ niñas, con una edad de 2 años y 1 mes a 7 años y 6 meses; el grupo 2 , con 13 pacientes, 4 (30.8\%) niños y 9 (69.2\%) niñas, con una edad de 2 años y 1 mes a 3 años y 11 meses; y el grupo 3 , con 28 pacientes, $12(42.9 \%)$ niños y $16(57.1 \%)$ niñas, con una edad de 4 años a 7 años y 6 meses.

Para el análisis de los datos se realizaron pruebas de estadística descriptiva con la finalidad de conocer el comportamiento de los datos con respecto a la media y las desviaciones estándar de las variables edad, tiempo de uso de los auxiliares, grupo de electrodos y unidades de corriente.

Para evaluar las uc necesarias en la calibración del implante coclear se construyeron ecuaciones de regresión múltiple para establecer una posible asociación entre las uc, la edad y el tiempo de uso del implante.
El modelo de regresión múltiple utilizado fue:

$\hat{y}=b_{0}+b_{1} x_{1}+b_{2} x_{2}$

donde:

$\hat{y}=$ valor estimado de la variable dependiente (uc).

$x_{1}, x_{2}=$ variables predictoras $\left(x_{1}=\right.$ tiempo de uso $\mathrm{y}$ $x_{2}=$ edad).

$b_{0} b_{1} b_{2}$ estimaciones muestrales de $\beta_{0}, \beta_{1}, \beta_{2}$,

Para el cálculo de las ecuaciones se utilizó el método de mínimos cuadrados:

$$
\begin{aligned}
& \sum y=b_{0^{n}}+b_{1} \sum x_{1}+b_{2} \sum x_{2} \\
& \sum y x_{1}=b_{0} \sum x_{1}+b_{1} \sum\left(x_{1}\right)^{2}+b_{2} \sum x_{1} x_{2} \\
& \sum y x_{2}=b_{0} \sum x_{2}+b_{1} \sum x_{1} x_{2}+b_{2} \sum\left(x_{2}\right)^{2}
\end{aligned}
$$

Para calcular el error estándar de la estimación se utilizó la fórmula:

$$
S_{y x s}=\sqrt{\frac{\sum(y-\hat{y})^{2}}{n-k}}
$$

donde:

$S_{y x s}=$ error estándar de la estimación.

$y=$ valores muestrales de (uc).

$\hat{y}=$ valores de calculados a partir del plano de regresión.

$k=$ número de parámetros linealmente independientes que deben estimarse (es el número de $b$, en la ecuación $k=3$ ).

$$
n=\text { tamaño de la muestra }\left(n_{1}=41, n_{2}=13, n_{3}=28\right) \text {. }
$$

\section{Resultados}

En el grupo 1, el promedio de edad fue de $4.54 \pm$ 1.36 años (media \pm desviación estándar), el índice de asimetría $\alpha_{3}$ fue de 0.511 , indicando una distribución ligeramente sesgada a la derecha debido al rango de edad obtenido por los sujetos estudiados (rango $=5.4$ ), y la media del tiempo de uso del implante coclear fue de $1.82 \pm 1.24$ años. Analizando las uc que se utilizaron para la calibración de los implantes de los pacientes de este grupo, se obtuvo que para el GE1 el promedio fue de $22.39 \pm 33.42$ uc, con un índice de asimetría de 1.94, indicando una distribución sesgada a la derecha definida por el rango de $125 \mathrm{uc}$; en el GE2, el promedio fue de $24.85 \pm 29.87$ uc, con un índice de asimetría de 1.651, distribución sesgada a la derecha y un rango de 115 uc; en el GE3, la media se ubicó en $19.32 \pm 21.6$ uc, con un índice de asimetría de 1.304, indicando una distribución sesgada a la derecha y un rango de 82 uc; y en el GE4, la media se ubicó en $20.2 \pm 22.21$ uc, con un índice de asimetría de 1.21, distribución sesgada a la derecha y rango de 90 uc. Se observa que el GE3 
Tabla 1. Estadísticos descriptivos del grupo 1

\begin{tabular}{|c|c|c|c|c|c|c|c|c|c|}
\hline & 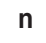 & Rango & Mínimo & Máximo & Media & $\mathrm{DE}$ & Varianza & Asimetría & Curtosis \\
\hline Edad & 41 & 5.4167 & 2.0833 & 7.5000 & 4.544685 & 1.364496 & 1.862 & 0.511 & -0.415 \\
\hline Tiempo de uso & 41 & 5.1667 & 0.0833 & 5.2500 & 1.827202 & 1.241694 & 1.542 & 0.597 & 0.289 \\
\hline uc GE1 & 41 & 125 & 0 & 125 & 22.39 & 33.426 & 1117.294 & 1.940 & 3.114 \\
\hline uc GE2 & 41 & 115 & 0 & 115 & 24.85 & 29.878 & 892.678 & 1.651 & 2.526 \\
\hline uc GE3 & 41 & 82 & 0 & 82 & 19.32 & 21.605 & 466.772 & 1.304 & 1.114 \\
\hline uc GE4 & 41 & 90 & 0 & 90 & 20.20 & 22.213 & 493.411 & 1.210 & 0.994 \\
\hline
\end{tabular}

DE: desviación estándar; GE: grupo de electrodos; uc: unidades de corriente.

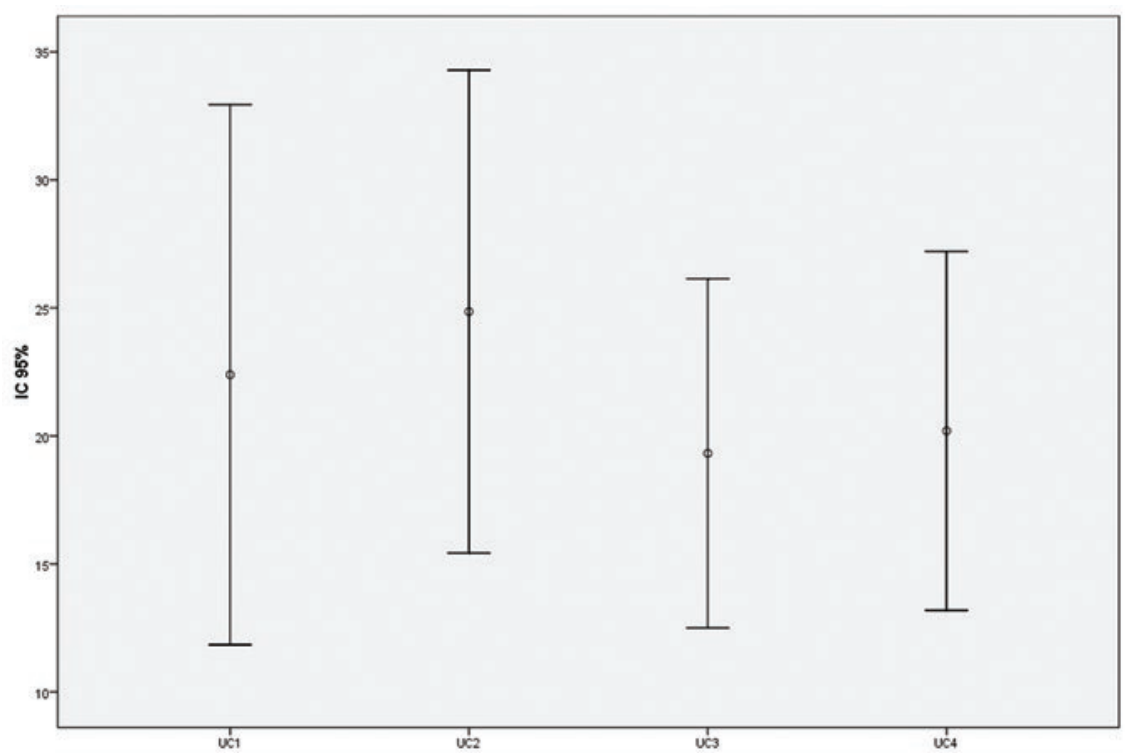

Figura 1. Gráfica de error de los cuatro grupos de electrodos del grupo 1. Se observa que GE3 y GE4 necesitaron menor cantidad de unidades de corriente (UC) para la calibración del implante coclear. IC 95\%: intervalo de confianza del 95\%.

y el GE4 presentan un similar índice de asimetría, con 1.304 y 1.21 uc respectivamente, con una diferencia entre ambos de 0.094 uc; así mismo, presentan similar comportamiento en el promedio y la desviación estándar al ser más pequeños en comparación con el GE1 y el GE2 (Tabla 1 y Fig. 1).

Al analizar los estadísticos descriptivos del grupo 2 ( $n=13$ ) se observa que el promedio de edad fue de $3.12 \pm 0.48$ años, el índice de asimetría $\alpha_{3}$ fue de -0.564 , indicando una distribución ligeramente sesgada a la izquierda debido al rango de edad obtenido por los sujetos estudiados (rango $=1.83$ ), y la media del tiempo de uso fue de $0.57 \pm 0.48$ años. Analizando las uc que se utilizaron para la calibración de los implantes de los pacientes de este grupo se obtuvo que, para el GE1, el promedio fue de $19 \pm 21.24$ uc, con un índice de asimetría de 1.014, indicando una distribución sesgada a la derecha definida por el rango de 60 uc; en el GE2, el promedio fue de $24.62 \pm 18.08$ uc, con un índice de asimetría de 0.187 , distribución sesgada ligeramente a la derecha y un rango de 55 uc; en el GE3, la media se ubicó en $21.54 \pm 16.25$ uc, con un índice de asimetría de 0.457 , indicando una distribución sesgada a la derecha y un rango de 50 uc; y en el GE4, la media fue de $25.46 \pm 18.03$ uc, con un índice de asimetría de 0.62 , con distribución sesgada a la derecha y rango de 55 uc. Se observa que el GE2 y el GE4 presentan similar índice de asimetría, con 0.187 y 0.062 uc respectivamente, con una diferencia entre ambos de 0.125 uc; así mismo, con respecto al promedio se observa que el GE1 presenta el más bajo (19) y la desviación estándar más amplia (21.24), mientas que el GE3 presenta el segundo promedio más bajo (21.54) y la dispersión más pequeña (16.25) (Tabla 2 y Fig. 2).

Al analizar los estadísticos descriptivos del grupo 3 $(n=28)$ se observa que el promedio de edad fue de $5.2 \pm 1.11$ años, el índice de asimetría $\alpha_{3}$ fue de 0.721 , 
Tabla 2. Estadísticos descriptivos del grupo 2

\begin{tabular}{|c|c|c|c|c|c|c|c|c|c|}
\hline & $\mathrm{N}$ & Rango & Mínimo & Máximo & Media & $\mathrm{DE}$ & Varianza & Asimetría & Curtosis \\
\hline Edad & 13 & 1.833 & 2.083 & 3.917 & 3.12817 & 0.484256 & 0.235 & -0.564 & 0.742 \\
\hline Tiempo de uso & 13 & 1.3333 & 0.0833 & 1.4166 & 0.576892 & 0.4828767 & 0.233 & 0.0447 & -1.082 \\
\hline uc GE1 & 13 & 60 & 0 & 60 & 19 & 21.241 & 451.167 & 1.014 & -0.216 \\
\hline uc GE2 & 13 & 55 & 0 & 55 & 24.62 & 18.081 & 326.923 & 0.187 & -0.399 \\
\hline uc GE3 & 13 & 50 & 0 & 50 & 21.54 & 16.256 & 264.269 & 0.457 & -0.813 \\
\hline uc GE4 & 13 & 55 & 0 & 55 & 25.4615 & 18.03522 & 325.269 & 0.062 & -1.207 \\
\hline
\end{tabular}

DE: desviación estándar; GE: grupo de electrodos; uc: unidades de corriente.

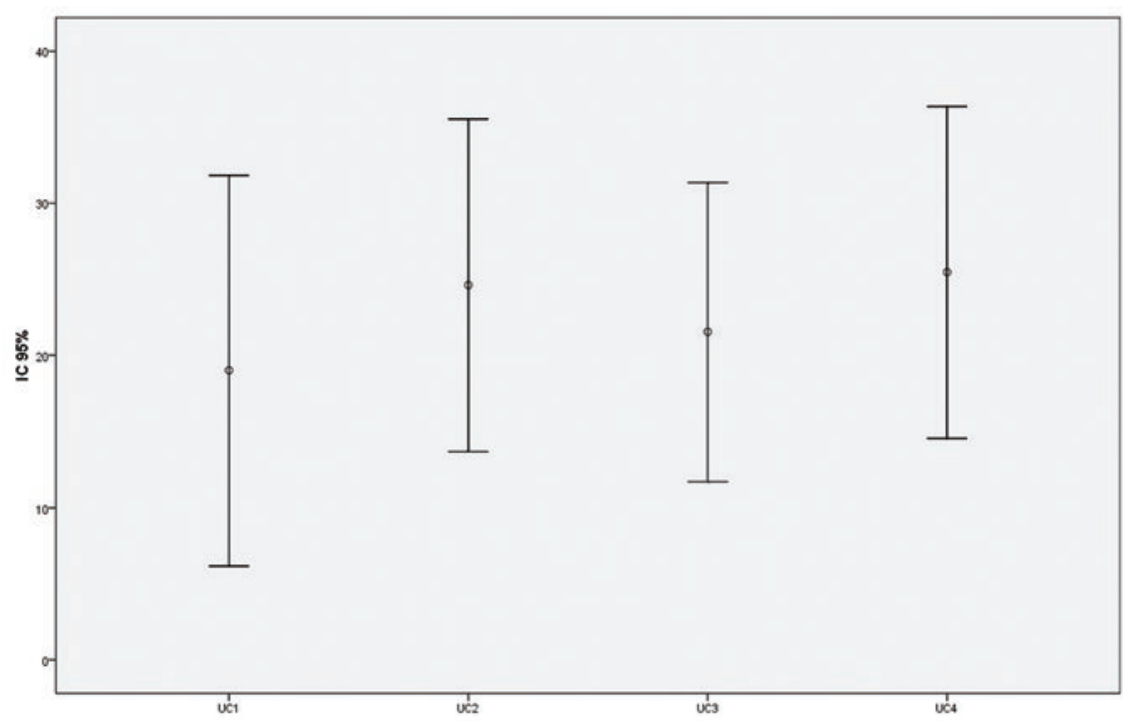

Figura 2. Gráfica de error de los cuatro grupos de electrodos del grupo 2. Se observa que los promedios en GE1 y GE3 presentaron valores más bajos que en GE2 y GE4. IC 95\%: intervalo de confianza del 95\%; UC: unidades de corriente.

indicando una distribución sesgada a la derecha debido al rango de edad obtenido por los sujetos estudiados (rango = 3.5), y la media del tiempo de uso fue de 2.4 \pm 1.03 años. Analizando las uc que se utilizaron para la calibración de los implante coclear de los pacientes de este grupo, se obtuvo que en el GE1 el promedio fue de $23.96 \pm 38.03$ uc, con un índice de asimetría de 1.834 , indicando una distribución sesgada a la derecha definida por el rango de $125 \mathrm{uc}$; en el GE2, el promedio fue de $24.96 \pm 34.31$ uc, con un índice de asimetría de 1.636, distribución sesgada a la derecha y un rango de 115 uc; en el GE3, la media se ubicó en $18.29 \pm 23.88$ uc, con un índice de asimetría de 1.484, indicando una distribución sesgada a la derecha y un rango de 82 uc; y en el GE4, la media fue de $17.75 \pm 23.8$ uc, con un índice de asimetría de 1.625, con distribución sesgada a la derecha y un rango de 90 uc. Se observa que el GE3 y el GE4 presentan similar desviación estándar, 23.887 y 23.809 uc respectivamente, con una diferencia entre ambos de 0.078 uc; así mismo, muestran similar comportamiento en el promedio y la desviación estándar al ser más pequeños en comparación con el GE1 y el GE2 (Tabla 3 y Fig. 3).

Se construyeron ecuaciones de regresión múltiple $x_{1}=$ tomando como variables tiempo de uso y $x_{2}=$ edad, con la finalidad de determinar las uc que se necesitaron en la calibración del implante coclear (y). De la misma manera, se calculó el error estándar de la estimación (Syxs) para conocer las desviaciones obtenidas en las ecuaciones.

Las ecuaciones calculadas para el grupo 1 fueron:

GE1 $\hat{y}=2.617-10.161 x_{1}+8.436 x_{2}$

GE2 $\hat{y}=18.505-7.535 x_{1}+4.426 x_{2}$

GE3 $\hat{y}=13.617-8.297 x_{1}+4.590 x_{2}$

GE4 $\hat{y}=12.727-7.510 x_{1}+4.663 x_{2}$

Las ecuaciones calculadas para el grupo 2 fueron:

GE1 $\hat{y}=67.296+4.427 x_{1}-16.256 x_{2}$

GE2 $\hat{y}=70.401+1.599 x_{1}-14.931 x_{2}$ 
Tabla 3. Estadísticos descriptivos del grupo 3

\begin{tabular}{|c|c|c|c|c|c|c|c|c|c|}
\hline & $\mathbf{n}$ & Rango & Mínimo & Máximo & Media & DE & Varianza & Asimetría & Curtosis \\
\hline Edad & 28 & 3.5 & 4 & 7.5 & 5.202354 & 1.1132955 & 1.239 & 0.721 & -0.730 \\
\hline Tiempo de uso & 28 & 4.5834 & 0.6666 & 5.25 & 2.407704 & 1.0384472 & 1.078 & .0807 & 1.309 \\
\hline uc GE1 & 28 & 125 & 0 & 125 & 23.96 & 38.035 & 1446.628 & 1.834 & 2.207 \\
\hline uc GE2 & 28 & 115 & 0 & 115 & 24.96 & 34.310 & 1177.147 & 1.636 & 1.8 \\
\hline uc GE3 & 28 & 82 & 0 & 82 & 18.29 & 23.887 & 570.582 & 1.484 & 1.296 \\
\hline uc GE4 & 28 & 90 & 0 & 90 & 17.75 & 23.809 & 566.861 & 1.625 & 2.032 \\
\hline
\end{tabular}

DE: desviación estándar; GE: grupo de electrodos; uc: unidades de corriente.

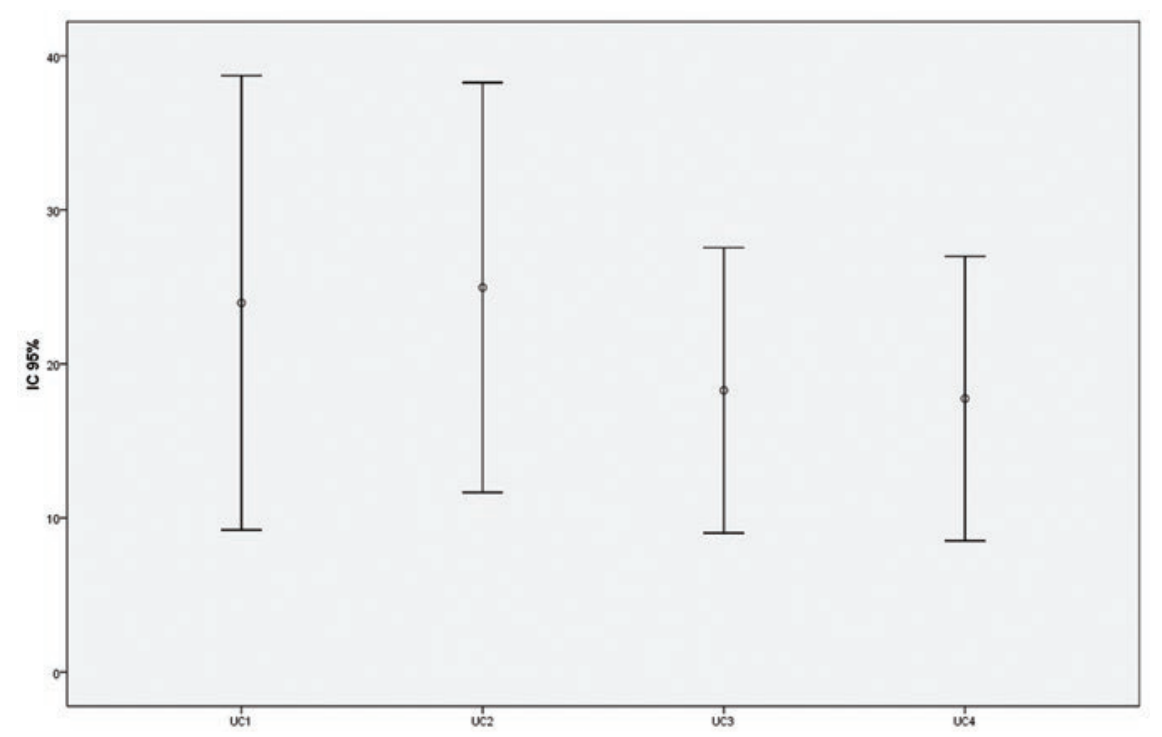

Figura 3. Gráfica de error de los cuatro grupos de electrodos del grupo 3. Se observa que los promedios obtenidos en GE1 y GE2 son mayores, y las barras de error de GE3 y GE4 son casi iguales. IC 95\%: intervalo de confianza del 95\%; UC: unidades de corriente.

GE3 $\hat{y}=83.329-8.894 x_{1}-18.113 x_{2}$

GE4 $\hat{y}=53.088-10.280 x_{1}-6.936 x_{2}$

$Y$ las ecuaciones calculadas para el grupo 3 fueron:

GE1 $\hat{y}=-0.371-18.8 x_{1}+13.379 x_{2}$

GE2 $\hat{y}=17.354-13.827 x_{1}+7.862 x_{2}$

GE3 $\hat{y}=5.657-12.244 x_{1}+8.094 x_{2}$

GE4 $\hat{y}=-4.452-7.772 x_{1}+7.865 x_{2}$

Donde $\hat{y}$ es el valor predictivo de las uc según $x_{1}=$ tiempo de uso y $x_{2}=$ edad.

Finalmente, se sustituyeron los valores originales de las variables tiempo de uso y edad para calcular el error por grupo de pacientes y por grupo de electrodos. De esta manera, en el grupo 1 se obtuvo una menor variabilidad en las uc en el GE3 y el GE4, con un error estándar de \pm 21.361 y \pm 22.22 respectivamente; en el grupo 2 se obtuvo el menor error estándar en el GE3, con \pm 13.761; y en el grupo 3, el menor error estándar se observó en el GE3 y el GE4, con \pm 23.453 y \pm 24.084 respectivamente (Tabla 4).

\section{Discusión}

Para la adquisición y el desarrollo del lenguaje se requiere la intervención de la citoarquitectura cerebral especializada en la codificación y la decodificación de sonidos que pertenecen a un sistema lingüístico, el óptimo funcionamiento del órgano auditivo, la integridad estructural y funcional del aparato fonoarticulador, la experiencia auditiva que involucra la plasticidad cerebral y la retroalimentación de sonidos procedentes del medio ambiente, generando cada uno de estos diferente tipo de información, siendo posiblemente el más importante el poder de la comunicación humana ${ }^{32}$.

Los pacientes con implante requieren tener la experiencia de los sonidos que provienen del medio ambiente, por lo que son necesarios tiempo y madurez neurofisiológica. Tal como se cita en la literatura, las calibraciones tras el implante en su mayoría están dadas por estímulo-respuesta del paciente cuando la edad lo permite ${ }^{5-9}$. 
Tabla 4. Error estándar de estimación por grupo de pacientes y grupo de electrodos

\begin{tabular}{lcccc}
\hline & Syxs GE1 & Syxs GE2 & Syxs GE3 & Syxs GE4 \\
\hline Grupo 1 & \pm 30.684 & \pm 30.207 & \pm 21.361 & \pm 22.220 \\
Grupo 2 & \pm 21.622 & \pm 18.189 & \pm 13.761 & \pm 18.474 \\
Grupo 3 & \pm 37.535 & \pm 34.366 & \pm 23.453 & \pm 24.084 \\
\hline
\end{tabular}

GE: grupo de electrodos.

En el presente trabajo se proponen ecuaciones matemáticas que pretenden establecer una correlación entre la edad biológica y el tiempo de uso del implante coclear con respecto a las uc necesarias para la calibración. El análisis de regresión múltiple multivariable es una nueva propuesta de análisis que no se ha reportado previamente en la literatura.

Raghunandhan, et al..$^{10}$ evidenciaron un incremento de las uc durante el proceso de ajuste del implante coclear, considerando exclusivamente la variable tiempo de uso. Reportaron al primer mes un promedio de 155 uc y al final del primer año un promedio de 272 uc. En nuestro estudio, las ecuaciones propuestas contemplan conjuntamente la edad del paciente, el tiempo de uso y el grupo de electrodos, determinando así los valores obtenidos para el error estándar de la estimación (Tabla 4).

En el presente trabajo, al evaluar el error estándar en cada ecuación por grupo encontramos que las uc necesarias varían según la madurez neurológica y la edad biológica del paciente. De esta manera, en el grupo $1(n=41)$ las dispersiones por grupo de electrodos fueron, para el GE1, \pm 30.684 ; para el GE2, \pm 30.207; para el GE3, \pm 21.361 ; y para el GE4, \pm 22.220 . En lo que respecta al grupo $2(n=13)$, para el GE1 fue \pm 21.622; para el GE2, \pm 18.189; para el GE3, \pm 13.761; y para el GE4, \pm 18.474. Finalmente, en el grupo $3(n=28)$, las uc para el GE1 fueron \pm 37.535 ; para el GE2, \pm 34.366 ; para el GE3, \pm 23.453 ; y para el GE4, \pm 24.084 . Se sugiere que el uso de estas ecuaciones puede ser confiable para el ajuste del implante coclear, ya que se calculan asociando tres variables (uc vs. edad y tiempo de implantación).

\section{Conclusiones}

Deben realizarse más trabajos de estas características ampliando el tamaño de la muestra con la finalidad de disminuir los errores estándar con respecto al tamaño de la población.

Las ecuaciones propuestas pueden ser de utilidad clínica al conocer las uc necesarias para el ajuste del implante coclear con respecto a la edad del paciente y el tiempo de uso del implante.

\section{Conflicto de intereses}

Los autores declaran que no existe conflicto de intereses.

\section{Responsabilidades éticas}

Protección de personas y animales. Los autores declaran que los procedimientos seguidos se conformaron a las normas éticas del comité de experimentación humana responsable y de acuerdo con la Asociación Médica Mundial y la Declaración de Helsinki.

Confidencialidad de los datos. Los autores declaran que han seguido los protocolos de su centro de trabajo sobre la publicación de datos de pacientes.

Derecho a la privacidad y consentimiento informado. Los autores han obtenido el consentimiento informado de los pacientes y/o sujetos referidos en el artículo. Este documento obra en poder del autor de correspondencia.

\section{Bibliografía}

1. Organización Mundial de la Salud. Sordera y pérdida de la audición. 2018. (Consultado el 26 de julio de 2018.) Disponible en: http://www. who.int/es/news-room/fact-sheets/detail/deafness-and-hearing-loss

2. Calderón-Leyva I, Díaz-Leines S, Arch-Tirado E, Lino-González AL. Analysis of the relationship between cognitive skills and unilateral sensory hearing loss. Neurologia. 2018;33:283-9.

3. Secretaría de Desarrollo Social. Diagnóstico sobre la situación de las personas con discapacidad en México. 2016. (Consultado el 21 de junio de 2018.) Disponible en: https://www.gob.mx/cms/uploads/attachment/ file/126572/Diagn_stico_sobre_la_Situaci_n_de_las_Personas_Con_ Discapacidad._Mayo_2016.pdf

4. National Institute on Deafness and Other Communication Disorders. Cochlear implants. 2017. (Consultado el 14 de julio de 2018.) Disponible en: https://www.nidcd.nih.gov/health/cochlear-implants

5. Banda RI, Castillo S, Roque G. Parámetros de programación del implante coclear. Bol Med Hosp Infant Mex. 2017;74:65-9.

6. Castro A, del Río L, Lassaletta L. Implantes cocleares y de tronco cerebral. En: Sociedad Española de Otorrinolaringología y Patología Cervico-Facial. Libro virtual de formación en ORL. 2014. Capítulo 34. p. 1-20. Disponible en: http://seorl.net/PDF/Otologia/034\%20-\%20IMPLANTES\%20COCLEARES\%20Y\%20DE\%20TRONCO.pdf

7. Kamal A. Comparison between behavioral, modified Brown ECAP, ESRT approaches in cochlear implants fitting in adults. 2017. (Consultado el 18 de mayo de 2018.) Disponible en: https://juniperpublishers.com/ ebooks/Comparison\%20Between \%20Behavioral,\%20Modified\%20 Brown\%20ECAP,\%20ESRTComparison\%20Between\%20Behavioral,\%20Modified\%20Brown\%20ECAP,\%20ESRT.pdf

8. Koci V, Stephan K. Early changes of electrical stapedius reflex threshold over time in patients supplied with $\mathrm{Cl}$. $8^{\text {th }}$ EFAS Congress $/ 10^{\text {th }}$ Congress of the German Society of Audiology. 2007. (Consultado el 15 de mayo de 2018.) Disponible en: http://www.uzh.ch/orl/dga2007/program/scientificprogram/Koci_V._Stephan_K.pdf

9. Bresnihan M, Norman G, Scott F, Viani L. Measurement of comfort levels by means of electrical stapedial reflex in children. Arch Otolaryngol Head Neck Surg. 2001;127:963-6.

10. Raghunandhan S, Ravikumar A, Kameswaran M, Mandke K, Ranjith R. Electrophysiological correlates of behavioral comfort levels in cochlear implantees: a prospective study. Indian J Otolaryngol Head Neck Surg. 2015;67:210-22. 
11. Fernández-Córdoba AC, Gutiérrez-Farfán IS, Chamlati-Aguirre LE, Alfaro-Rodríguez A, Durand-Rivera A. Modificación de umbrales T en pacientes con implante coclear como una alternativa de programación en relación con el tiempo. Rev Invest Clin. 2014;66:247-51.

12. Shapiro WH, Bradham TS. Cochlear implant programming. Otolaryngol Clin North Am. 2012;45:111-27.

13. Zwolan TA, O'Sullivan MB, Fink NE, Niparko JK; CDACI Investigative Team. Electric charge requirements of pediatric cochlear implant recipients enrolled in the Childhood Development After Cochlear Implantation study. Otol Neurotol. 2008;29:143-8.

14. Zeng FG, Rebscher S, Harrison W, Sun X, Feng H. Cochlear implants: system design, integration, and evaluation. IEEE Rev Biomed Eng. 2008:1:115-42

15. Moore BC. Coding of sounds in the auditory system and its relevance to signal processing and coding in cochlear implants. Otol Neurotol. 2003;24:243-54.

16. Andrade KC, Leal M de C, Muniz LF, Menezes P de L, Albuquerque KM Carnaúba AT. The importance of electrically evoked stapedial reflex in cochlear implant. Braz J Otorhinolaryngol. 2014;80:68-77.

17. Vaerenberg B, Smits C, De Ceulaer G, Zir E, Harman S, Jaspers N, et al. Cochlear implant programming: a global survey on the state of the art Scientific World Journal. 2014:(501738):1-13

18. Raghunandhan S. Clinical correlation of multimodality electrophysiological assessment with psychophysical behavioural responses in cochlear implants. Chapter 3. 2015. (Consultado el 10 de julio de 2018.) Disponible en: http://shodhganga.inflibnet.ac.in:8080/jspui/handle/10603/37469

19. Arias $P$, Hernández $M C$, Zuluaga $P$, Calero $P$. Método de evaluación en campo libre de los umbrales en pacientes con implante coclear: utilidad de los potenciales evocados auditivos de estado estable para la valoración del rendimiento objetivo de los implantes cocleares. Auditio: Rev Electr Audiol. 2015;4:47-52.

20. Caner G, Olgun L, Gültekin G, Balaban M. Optimizing fitting in children using objective measures such as neural response imaging and electrically evoked stapedius reflex threshold. Otol Neurotol. 2007;28:637-40.

21. Walkowiak A, Lorens A, Kostek B, Skarzynski H, Polak M. ESRT, ART, and $\mathrm{MCL}$ correlations in experienced paediatric cochlear implant users. Cochlear Implants Int. 2010;11:S482-4.
22. Advanced Bionics ${ }^{\circledast}$ Corporation. Auditory Research Bulletin Interim Edition 2006. (Consultado el 17 de julio de 2018.) Disponible en: https:// advancedbionics.com/content/dam/advancedbionics/Documents/Global/ en_ce/Professional/AB-Studies-and-Research/Auditory-Research-Bulletins/AB Auditory Research Bulletin Interim Report 2006.pdf

23. Christov F, Munder P, Berg L, Bagus $\bar{H}$, Lang $\bar{S}$, Arweiler-Harbeck D. ECAP analysis in cochlear implant patients as a function of patient's age and electrode-design. Eur Ann Otorhinolaryngol Head Neck Dis. 2016;133:S1-3.

24. Gordon KA, Papsin BC, Harrison RV. Toward a battery of behavioral and objective measures to achieve optimal cochlear implant stimulation levels in children. Ear Hear. 2004;25:447-63.

25. Kosaner J, Spitzer P, Bayguzina S, Gultekin M, Behar LA. Comparing eSRT and eCAP measurements in pediatric MED-EL cochlear implant users. Cochlear Implants Int. 2018;19:153-61.

26. Buckler L, Dawson K, Overstreet E. Relationship between electrical stapedial reflex thresholds and HiRes ${ }^{\mathrm{TM}}$ program settings: potential tool for pediatric cochlear-implant fitting. 2003. (Consultado el 16 de julio de 2018.) Disponible en: https://advancedbionics.com/content/dam/advancedbionics/ Documents/Regional/BR/Relationship between_ESRT and HiRes.pdf

27. Walkowiak A, Lorens A, Polak M, Kostek B, Skarzynski H, Szkielkowska A, et al. Evoked stapedius reflex and compound action potential thresholds versus most comfortable loudness level: assessment of their relation for charge-based fitting strategies in implant users. ORL J Otorhinolaryngol Relat Spec. 2011;73:189-95

28. Mittal R, Panwar SS, Nair S, Sinha VR, Ramesh AV. Mapping of paediatric cochlear implant recipients using EABR as a tool. J Otol Rhinol. 2015;21:14-8.

29. Manrique Rodríguez M, Huarte Irujo A. Organisation of a cochlear implant programme. Acta Otorrinolaringol Esp. 2013;64:55-67.

30. Guenser G, Laudanski J, Phillipon B, Backus BC, Bordure P, Romanet P, et al. The relationship between electrical auditory brainstem responses and perceptual thresholds in Digisonic ${ }^{\circledR}$ SP cochlear implant users. Cochlear Implants Int. 2015;16:32-8.

31. Lorens A, Walkowiak A, Piotrowska A, Skarzynski H, Anderson I. ESRT and MCL correlations in experienced paediatric cochlear implant users. Cochlear Implants Int. 2004;5:28-37.

32. Martínez-Beneyto P, Morant A, Pitarch MI Latorre E, Platero A, Marco J. La implantación coclear pediátrica en el periodo crítico de la vía auditiva, nuestra experiencia. Acta Otorrinolaringol Esp. 2009;60:311-7. 